\title{
CARACTERIZACIÓN ESTRUCTURAL DE MADERA ASERRADA DE PINOS CULTIVADOS EN URUGUAY
}

\author{
STRUCTURAL CHARACTERIZATION OF PINE LUMBER \\ FROM URUGUAY
}

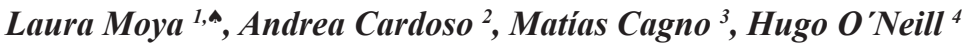

\begin{abstract}
RESUMEN
La disponibilidad de madera aserrada producida en Uruguay ha tenido un incremento significativo en los últimos treinta años debido a la política gubernamental para promover plantaciones de rápido crecimiento. Para aplicaciones estructurales, la madera debe estar clasificada según su resistencia mecánica, rigidez y densidad, y sus propiedades disponibles para profesionales y técnicos. Este trabajo estuvo motivado por la falta de madera clasificada y de un sistema de clases resistentes en Uruguay. El objetivo fue caracterizar mecánicamente madera aserrada de Pinus elliottii y P. taeda y asociar sus propiedades a grados estructurales. El estudio evaluó una muestra representativa del material que se produce y comercializa en Uruguay, que incluyó madera de 25 años del litoral y de 15 años del suroeste. Aproximadamente 900 piezas de tamaño estructural previamente clasificadas en tres grados cualitativos fueron ensayadas en flexión, compresión y tracción-paralela a la fibra. Cada grado fue asociado a una familia de tensiones y comparado con los valores de EN 338 y NCh 1198. El grupo superior con piezas de 25 años del litoral pudo ser asignado a la clase $\mathrm{C} 14$, mientras que ninguno a las exigencias de $\mathrm{NCh}$ 1198. Ningún grupo de 15 años del suroeste cumplió con los requisitos estructurales internacionales.
\end{abstract}

Palabras clave: Grados estructurales, madera estructural, Pinus elliottii, Pinus taeda.

\begin{abstract}
The availability of local produced lumber has significantly increased in the last thirty years in Uruguay due to a governmental policy to promote fast-grown plantations. For structural applications lumber must be strength graded and the properties should be available for professionals and customers. This research was motivated by the lack of strength-graded lumber and a strength class system in Uruguay. The objective was to characterize Pinus elliottii and Pinus taeda timber and to assign strength grades. The study evaluated a representative sample of currently produced material in Uruguay, including lumber from a 25-year-old west and 15-year- old southern-west plantations. Approximately 900 structural pieces were graded in three qualitative grades, and then tested in bending, compression- and tensile- parallel to grain. For each grade, a group of strength properties was associated. Comparison with EN 338 strength classes and NCh 1198 indicated that the superior group comprised of 25-year-old timber could be assigned to C14 class, while none complied with NCh 1198 requirements. None of the 15 year-old groups attained the international structural requirements.
\end{abstract}

Keywords: Pinus elliottii, Pinus taeda, strength grades, structural timber.

\footnotetext{
Profesor asociado. Facultad de Arquitectura. Universidad ORT Uruguay. Montevideo. Uruguay. moya@ort.edu.uy

${ }^{2}$ Consultor junior. Gerencia de I+D+i. Laboratorio Tecnológico del Uruguay. Montevideo. Uruguay. acardoso@latu.org.uy

${ }^{3}$ Consultor junior. Gerencia de I+D+i. Laboratorio Tecnológico del Uruguay. Montevideo. Uruguay. mcagno@latu.org.uy

${ }^{4}$ Investigador senior. Gerencia de I+D+i. Laboratorio Tecnológico del Uruguay. Montevideo. Uruguay. honeill@latu.org.uy

^Autor para correspondencia: moya@ort.edu.uy

Recibido: 04.04.2014 Aceptado: 22. 01. 2015
} 


\section{INTRODUCCIÓN}

En Uruguay el sector forestal ha tenido un incremento significativo en los últimos treinta años como consecuencia de la política gubernamental para promover las plantaciones forestales. En la década de los ochenta, estimaciones de la Dirección General Forestal del Ministerio de Ganadería Agricultura y Pesca (MGAP) indicaban la existencia de entre 400000 y 600000 ha ocupadas por especies nativas de árboles de pequeño diámetro $(15-20 \mathrm{~cm})$ de escaso uso industrial, aproximadamente $120000 \mathrm{a} 137000 \mathrm{ha}$ de Pinus y Eucalyptus, y 70000 ha compuestas por otras especies exóticas (MGAP 2005). Actualmente las plantaciones forestales cubren aproximadamente un millón de ha, de las cuales el 25 por ciento del total de la superficie plantada corresponde a Pinus $s p$. y dentro del género, las especies $P$. elliottii y $P$. taeda para madera aserrada y productos de ingeniería de madera comprenden el 39 y 45 por ciento respectivamente (MGAP 2010). Debido a la similitud de sus propiedades físicas, estas dos especies usualmente son plantadas y se comercializan en el mercado en forma mezclada. Parte de esta madera podría ser empleada en programas de viviendas y responder parcialmente a la demanda de 60000-100000 viviendas de interés social (Montevideo Portal 2013). La incorporación de tecnologías de construcción con madera en la industria de la construcción permitiría además reducir tiempos y costos de ejecución, como en el plan piloto de cuatro viviendas de $60 \mathrm{~m}^{2}$ cada una con estructura de madera realizado en la ciudad de Rivera en 6 semanas (MVOTMA 2011). Pese a esta experiencia y otras exitosas en el país, la madera en general y la de pinos en particular, sigue siendo empleada escasamente para fines estructurales. Entre los factores que explican la no-adopción del material, destacan los prejuicios culturales, el desconocimiento por parte de usuarios y técnicos de las ventajas del material, y principalmente la falta de madera clasificada de acuerdo a su resistencia y rigidez en el mercado local. A nivel internacional, existen varias normas de clasificación visual y mecánica y una tendencia creciente hacia la utilización de sistemas de clases resistentes como el establecido en la norma europea EN 338 (2010), o el de grados estructurales de la norma chilena NCh 1198 (2006).

A nivel nacional, desde mediados de la década de los noventa el Departamento de Proyectos Forestales del Laboratorio Tecnológico del Uruguay (LATU) viene trabajando en forma independiente, y asociativa con varios actores del sector forestal maderero y la academia en la caracterización de maderas producidas en Uruguay. Cuenta con una extensa base de datos parte de la cual ha sido analizada y publicada en los Informes $\mathrm{N}^{\circ} 1$ y No 2 del Grupo Técnico de Madera Aserrada de Pino GT3 (O'Neill et al. 2002, 2003), Notas técnicas (O’Neill et al. 2003, O’Neill 2004) e Informes de investigación (Pérez Favaro et al. 2000). En un trabajo pionero de aplicación de normas de clasificación visual, Pérez del Castillo (2001) estudió 80 vigas de Pinus elliottii de 43 años del sur y encontró que el 75 por ciento de las vigas cumplieron con los requisitos para uso en la construcción establecidos en la norma JAS 143 (1991). En un segundo trabajo Pérez del Castillo et al. (2003), aplicaron la norma NCh 1207 (1990) a 188 piezas de madera de Pinus elliottii y P. taeda de 24 años del litoral y observaron que el 84 por ciento de las piezas extraídas de la zona cercana a la corteza cumplieron con los requisitos del grado superior de la norma chilena, y el noventa y dos por ciento de las que contenían médula no calificaron estructuralmente. Por otro lado, O'Neill et al. (2002), (2003), estudiaron piezas de madera libre de médula provenientes de la primera y segunda troza de Pinus elliottii y P. taeda de 24 años del litoral y observaron valores de rigidez, tensión de rotura y densidad concordantes con los publicados en la literatura internacional para las mismas especies. Los trabajos mencionados han focalizado el estudio en piezas extraídas de trozas especialmente seleccionadas y preparadas en laboratorio. Sin embargo, ninguno ha establecido un procedimiento para determinar las propiedades de diseño y asociarlas a grados estructurales para la madera aserrada de pinos nacionales que se comercializa en Uruguay. El objetivo general de este estudio consistió en caracterizar mecánicamente madera aserrada de Pinus elliottii y P. taeda y asociar sus propiedades a grados estructurales, mediante la aplicación de la norma de clasificación visual $\mathrm{NCh}$ 1207 (2005). 


\section{MATERIALES Y MÉTODOS}

\section{Características de la muestra y clasificación de tablas}

Con la finalidad de obtener material representativo de la producción nacional, dos muestreos provenientes de plantaciones y aserraderos de dos empresas que comercializan madera en el país fueron estudiados: M1, localizado en el departamento de Paysandú (litoral), compuesto por árboles de 25 años de edad y de diámetros a altura de pecho (DAP) de $450 \mathrm{~mm}$ (promedio), y M2, ubicado en el departamento de San José (suroeste), con árboles de 15 años y de $300 \mathrm{~mm}$ de DAP. La composición de especies, similar en ambos muestreos, consistió en $90 \%$ de $P$. elliottii y $10 \%$ de $P$. taeda. El marco de plantación fue de $3 \mathrm{~m}$ x $3 \mathrm{~m}$, y $4 \mathrm{~m}$ x 2,50 m en M1 y M2 respectivamente. En ambos montes se realizaron raleos a los 5, 7 y 10 años, y en M1 adicionalmente a los 15 años. De la línea de producción de cada aserradero, se seleccionaron de un total de más de 600,480 piezas de $60 \mathrm{~mm}$ x $170 \mathrm{~mm}$ x $3300 \mathrm{~mm}$, totalizando 960 unidades entre ambos muestreos. Mediante evaluación visual de nudosidades y presencia de médula, las piezas fueron pre-clasificadas in situ simplificando los lineamientos de NCh 1207 (2005), en tres grados: grado selecto (GS), grado 1 (G1) y grado 2 (G2). Las cabezas de las piezas fueron pintadas a efectos de identificar su grado. Todas las piezas de M1 y M2 fueron secadas en el mismo horno y con igual agenda hasta alcanzar aproximadamente $18 \%$ de contenido de humedad $(\mathrm{CH})$; luego fueron cepilladas en sus cuatro caras hasta obtener dimensiones de 50 x 150 × $3200 \mathrm{~mm}$, embaladas en film de polietileno y trasladadas al Departamento de Proyectos Forestales del LATU. Allí fueron clasificadas en segunda instancia siguiendo las especificaciones de NCh 1207 (2005). Primero se evaluaron alabeos, atribuibles principalmente al proceso de secado, que determinaron el rechazo o la aceptación de la pieza. Consecutivamente se realizó la clasificación por nudosidades, y finalmente se valoraron otros atributos, como inclinación de la fibra, arista faltante, bolsillos de resina y corteza, fisuras y médula.

\section{Ensayos en el grado}

Luego de clasificadas, el conjunto de piezas fue dividido en tres grupos para ensayos de flexión, compresión y tracción paralela a la fibra. Cada grupo contó con una cantidad aproximadamente igual de piezas de cada grado.

Las piezas destinadas a ensayos de compresión y tracción fueron embaladas en film de polietileno y enviadas al Instituto Forestal INFOR de Concepción, Chile, donde se realizaron los ensayos correspondientes.

Los ensayos de flexión fueron realizados en el Departamento de Proyectos Forestales del LATU, según ASTM D198-09 (2011) con la disposición de la viga simplemente apoyada y la aplicación de dos cargas en los extremos del tercio central. Se utilizó una máquina universal Minebea con una celda de carga de $250 \mathrm{kN}$ y con capacidad para aplicar cargas a velocidad constante. El vuelco o pandeo lateral durante el ensayo fue impedido mediante dos soportes laterales, cuyas características permitieron el movimiento vertical y evitar la fricción de la viga, cada uno ubicado entre el punto de aplicación de la carga y la reacción. La deformación fue medida con un extensómetro ubicado en el plano neutro y en la mitad de la luz de la pieza. Para cada viga se calculó la tensión de rotura $\left(f_{m}\right)$ y el módulo de elasticidad global en flexión $\left(E_{m, g}\right)$ de acuerdo a:

$$
\begin{aligned}
f_{m} & =\frac{F_{m a \dot{x}} L}{b h^{2}} \\
E_{m, g} & =\frac{L^{3}\left(F_{2}-F_{1}\right)}{4,7 b h^{3}\left(\omega_{2}-\omega_{1}\right)}
\end{aligned}
$$

donde $F_{\text {máx }}$ es el valor de la carga máxima aplicada; $L$ es la luz de ensayo $(2700 \mathrm{~mm}) ; b$ y $h$ son base y altura respectivamente de la sección transversal; $F_{2}-F_{1}$ incremento de carga en el tramo recto de la curva carga deformación; $\omega_{2}-\omega_{1}$ incremento de la deformación correspondiente a $F_{2}-F_{1}$. 
Adicionalmente, el módulo de elasticidad local o libre de corte $\left(E_{m, l}\right)$ fue derivado a partir del global de acuerdo a (ASTM D198-09 2011):

$$
E_{m, l}=\frac{E_{m g}}{\left(1-\frac{l\left(F_{2}-F_{1}\right.}{5 b h G\left(\omega_{2}-\omega_{1}\right.}\right)}
$$

donde $G$ es el módulo de corte, estimado de acuerdo a (Bodig et al. 1993):

$$
G=\frac{E_{m, g}}{16}
$$

Luego de finalizado el ensayo, de cada viga se extrajo una probeta de dimensiones iguales a la sección transversal para determinar la densidad aparente corriente y el CH de acuerdo a ASTM D 2395-07 (2011).

Los ensayos de compresión paralela fueron realizados de acuerdo a NCh 3028/1 (2006), en una máquina de ensayo con un marco de carga/reacción de compresión diseñado para una capacidad de $400 \mathrm{kN}$, con un cilindro hidráulico marca Enerpac de $300 \mathrm{kN}$ de capacidad. La adquisición de datos se realizó en forma digital directa a PC mediante un transductor de presión de $60 \mathrm{MPa}$ de capacidad y $6,9 \mathrm{kPa}$ de sensibilidad, que permite el registro de datos en forma continua con frecuencias superiores a 100 datos por segundo. Para cada pieza fue determinada la tensión de rotura de compresión $\left(f_{c, 0}\right)$. Luego de finalizado el ensayo, de cada pieza se extrajo una probeta de dimensiones iguales a la sección transversal para determinar la densidad aparente corriente y el CH de acuerdo a NCh 3028/1 (2006).

Los ensayos de tracción paralela a la fibra fueron realizados de acuerdo a NCh 3028/1 (2006), en una máquina de ensayo Metriguard 403, con una celda de carga de $500 \mathrm{kN}$, con un sistema compuesto por dos cilindros hidráulicos marca Enerpac de $500 \mathrm{kN}$ de capacidad. La adquisición de datos se realizó en forma digital directa a PC mediante un transductor de presión de $60 \mathrm{MPa}$ de capacidad y $6,9 \mathrm{kPa}$ de sensibilidad. Para cada pieza fue determinada la tensión de rotura de tracción $\left(f_{t, 0}\right)$. Luego de finalizado el ensayo, de cada pieza se extrajo una probeta de dimensiones iguales a la sección transversal para determinar la densidad aparente corriente y el CH de acuerdo a NCh 3028/1 (2006). 


\section{Ajuste de las propiedades mecánicas y densidad por contenido de humedad}

Los valores experimentales de resistencias de flexión, compresión paralela y tracción paralela a la fibra que superaron los umbrales de 16,6 9,65 y 21,7 MPa respectivamente, fueron ajustados al 12\% de CH de acuerdo a (ASTM D 1990-07 2011):

$$
S_{2}=S_{1}+\left\{\frac{\left(S_{1}-B_{1}\right)}{\left(B_{2}-M_{1}\right.}\right\}\left(M_{1}-M_{2}\right)
$$

donde $\mathrm{S}_{1}$ y $\mathrm{S}_{2}$ son las propiedades (en $\mathrm{MPa}$ ) determinadas al $\mathrm{CH} \mathrm{M}_{1}$ y $\mathrm{M}_{2}$ (en \%) respectivamente; $\mathrm{B}_{1}$ y $\mathrm{B}_{2}$ son constantes de humedad.

Los módulos de elasticidad fueron ajustados al 12\% de CH de acuerdo a (ASTM D1990-07 2011):

$$
S_{2}=S_{1} \frac{\left\lfloor B_{1}-\left(B_{2} \times M_{2}\right)\right\rfloor}{\left[B_{1}-\left(B_{2} \times M_{1}\right)\right]}
$$

donde $\mathrm{S}_{1}$ y $\mathrm{S}_{2}$ son las propiedades (en $\mathrm{MPa}$ ) determinadas al $\mathrm{CH}_{1}$ y $\mathrm{M}_{2}$ (en \%) respectivamente; $\mathrm{B}_{1}$ y $\mathrm{B}_{2}$ son constantes de humedad.

La densidad fue ajustada al 12\% de CH de acuerdo a (USDA1999):

$$
D_{2}=\frac{D_{1}}{\left[1-0,265\left(30-M_{2}\right) / 100 D_{1}\right]}
$$
verde).

donde $\mathrm{D}_{2}$ es la densidad al $\mathrm{CH}$ deseado $\mathrm{M}_{2}$ (en \%); $\mathrm{D}_{1}$ es la densidad básica (peso seco y volumen

\section{Análisis de datos y derivación de tensiones}

La asignación a grados estructurales se efectuó para calidades visuales por lo cual la metodología de clasificación fue contrastada con los resultados de los ensayos mecánicos. Estudios previos (Moya et al. 2013) realizados sobre cuerpos de prueba de pequeñas dimensiones y libre de defectos mostraron diferencias significativas entre la madera de los muestreos M1 y M2. Esta observación fue verificada en el presente estudio para piezas de tamaño estructural mediante análisis de varianza (ANOVA). Adicionalmente dentro de cada muestreo, las propiedades estructurales fueron sujetas primero a ANOVA para rechazar la hipótesis nula de igualdad de medias de los grados, y luego al test Fisher LSD (Least Significant Difference) para comparar las medias y establecer diferencias entre pares. 
El procedimiento de derivación de tensiones de los grados estructurales se realizó de acuerdo a ASTM D 2915-10 (2011), limitando el percentil de exclusión al 5\% y con una confiabilidad estadística recomendada para piezas estructurales del $75 \%$. Para cada grupo, los datos fueron ordenados en orden creciente y la estadística descriptiva estimada. Para cada propiedad el valor característico $\left(f_{k}\right)$ quedó definido por el valor de ensayo asociado a la estadística de orden que corresponde al 5 por ciento del tamaño de la muestra. Cuando este valor no coincidió con un resultado experimental, se realizó interpolación lineal entre los dos valores más cercanos. Vale señalar que si bien en el presente estudio fueron utilizados los cuerpos normativos de INN y ASTM, las normas chilenas NCh 3028/1 y NCh 3028/2, especifican lineamientos de ensayos y criterios de análisis similares a los indicados en ASTM D 198, ASTM D 1990, y ASTM D 2915, y los datos presentados en este artículo son consistentes con ambos corpus. Adicionalmente, a efectos de incluir las piezas de madera en un determinado grado estructural del sistema chileno para pino radiata, las propiedades tabuladas en NCh 1198 (2005) fueron empleadas para derivar sus correspondientes valores característicos de resistencias de flexión, tracción paralela y compresión paralela, que ajustados al igual que el valor medio del módulo de elasticidad global a piezas de $150 \mathrm{~mm}$ de altura, permitieron realizar una justa comparación.

Por otro lado, se evaluó la posibilidad de incluir las piezas estudiadas en una determinada clase resistente del sistema europeo; los datos de las propiedades estructurales fueron referidos y eventualmente ajustados a las condiciones establecidas en EN 384 (2010) y EN 408 (2010), y luego de procesados en grupos de tensiones, comparados con las clases de EN 338.

\section{RESULTADOS Y DISCUSIÓN}

La clasificación visual realizada en laboratorio confirmó al 80\% de las piezas en sus grados iniciales (GS, G1 o G2), asignó a otro distinto al inicial al 7\% y rechazó (R), fundamentalmente por alabeos, al $11 \%$ (Tabla 1).

Tabla 1. Cantidad de piezas clasificadas de acuerdo a NCh 1207.

\begin{tabular}{cc}
\hline Grado & Cantidad \\
\hline GS & 293 \\
G1 & 292 \\
G2 & 255 \\
R & 111 \\
Total & 951 \\
\hline
\end{tabular}

\section{Diferencias entre muestreos}

Los resultados de ANOVA sintetizados en la tabla 2 mostraron diferencias significativas en el comportamiento mecánico de las piezas de los muestreos M1 y M2, y confirmaron lo reportado por Moya et al. (2013), en su estudio de pequeñas probetas libres de defectos. En ese trabajo se sugiere que las diferencias entre propiedades de la madera de 24 años del litoral y la de 15 años del suroeste, son atribuibles principalmente a la excesiva proporción de madera juvenil presente en esta última. La constatación de esas diferencias entre muestreos llevó a continuar el análisis en forma independiente uno del otro. 
Tabla 2. Análisis de varianza de propiedades mecánicas de cuerpos de prueba de Pinus taeda y P. elliottii.

\begin{tabular}{cccccc}
\hline \multicolumn{5}{c}{ Valores de p- M1 vs M2 ${ }^{1}$ para las propiedades: } \\
\hline$E_{m, g}$ & $E_{m, l}$ & $f_{m}$ & $f_{c, 0}$ & $f_{t, 0}$ & $\rho$ \\
$\mathrm{p}<0,001$ & $\mathrm{p}<0,001$ & $\mathrm{p}<0,001$ & $\mathrm{p}<0,001$ & $\mathrm{p}<0,001$ & $\mathrm{p}<0,001$ \\
\hline
\end{tabular}

${ }^{\mathrm{l}} \mathrm{M} 1$ y M2: muestreos constituidos por madera de árboles de 25 años del litoral, y de 15 años del suroeste respectivamente

La tabla 3 muestra los resultados de los análisis estadísticos de las propiedades de flexión y densidad de los cuerpos de prueba de los muestreos M1 y M2. Los valores medios de módulo de elasticidad global $\left(E_{m, g}\right)$, resistencia de flexión $\left(f_{m}\right)$ y densidad $(\rho)$ de las vigas de $\mathrm{M}_{1}$, se situaron entre $5500-7400$ $\mathrm{MPa}, 23-47 \mathrm{MPa}$, y $410-470 \mathrm{~kg} / \mathrm{m}^{3}$, respectivamente, y son consistentes con los valores publicados en la literatura nacional ( $\mathrm{O}^{\prime} \mathrm{N}$ eill et al. 2002, 2003). Para M2, los valores variaron entre $4600-5600$ MPa, 20-29 MPa y 360-380 kg/m³ , resultados que son similares a los reportados por Biblis (2006) en su estudio sobre piezas de madera de Pinus taeda de 19 años. En todos los casos, el CH de las probetas extraídas de los cuerpos de prueba de tamaño estructural luego de los respectivos ensayos de flexión, compresión y tracción, se situó entre 10 y $14 \%$.

En M1 y M2, el patrón de falla de rotura en flexión se inició en las zonas cercanas a los nudos, al igual que lo observado por Pérez del Castillo et al. (2003).

Dentro de cada muestreo ANOVA rechazó la hipótesis nula de igualdad de medias de las propiedades de flexión y densidad. Cuando se realizó la comparación pareada (entre grados) para las propiedades de las piezas de M1, el test de Fisher LSD encontró que la rigidez y la densidad de GS son significativamente mayores que las respectivas de $\mathrm{G} 1$ y G2, no habiendo detectado diferencias entre los dos grados inferiores; mientras que para la resistencia de flexión, se encontraron diferencias entre los tres grados.

Análogamente para las piezas de M2, LSD detectó que la rigidez y la densidad de GS son significativamente mayores que las respectivas de G2, mientras que la resistencia de flexión de las piezas G2 es significativamente inferior que las resistencias de G1 y GS.

Tabla 3. Análisis estadístico de propiedades de flexión y densidad ${ }^{1}$.

\begin{tabular}{ccccccccccc}
\hline Muestreo $^{2}$ & Grado & $\begin{array}{c}\mathrm{N}^{0} \\
\text { rep }\end{array}$ & $\begin{array}{c}E_{m, g}^{3} \\
{[\mathrm{MPa}]}\end{array}$ & $\mathrm{LSD}^{4}$ & $\begin{array}{c}E_{m, l}{ }^{3} \\
{[\mathrm{MPa}]}\end{array}$ & $\mathrm{LSD}^{4}$ & $\begin{array}{c}f_{m}^{3} \\
{[\mathrm{MPa}]}\end{array}$ & $\mathrm{LSD}^{4}$ & $\begin{array}{c}\rho^{3} \\
{\left[\mathrm{~kg} / \mathrm{m}^{3}\right]}\end{array}$ & $\mathrm{SD}^{4}$ \\
\hline \multirow{3}{*}{$\mathrm{M} 1$} & GS & 55 & $7489(2370)$ & $\mathrm{a}$ & $7852(2484)$ & $\mathrm{a}$ & $47(15)$ & $\mathrm{a}$ & $470(43)$ & $\mathrm{a}$ \\
& $\mathrm{G} 1$ & 31 & $5940(2587)$ & $\mathrm{b}$ & $6231(2714)$ & $\mathrm{b}$ & $31(12)$ & $\mathrm{b}$ & $423(23)$ & $\mathrm{b}$ \\
& $\mathrm{G} 2$ & 16 & $5594(1549)$ & $\mathrm{b}$ & $5869(1622)$ & $\mathrm{b}$ & $23(10)$ & $\mathrm{c}$ & $414(167)$ & $\mathrm{b}$ \\
\hline \multirow{2}{*}{$\mathrm{M} 2$} & GS & 29 & $5675(1545)$ & $\mathrm{a}$ & $5955(1419)$ & $\mathrm{a}$ & $29(11)$ & $\mathrm{a}$ & $378(33)$ & $\mathrm{a}$ \\
& $\mathrm{G} 1$ & 76 & $4960(1211)$ & $\mathrm{ab}$ & $5207(1270)$ & $\mathrm{ab}$ & $26(8)$ & $\mathrm{a}$ & $368(38)$ & $\mathrm{ab}$ \\
& $\mathrm{G} 2$ & 44 & $4681(1199)$ & $\mathrm{b}$ & $4915(1258)$ & $\mathrm{b}$ & $20(9)$ & $\mathrm{b}$ & $360(21)$ & $\mathrm{b}$ \\
\hline
\end{tabular}

\footnotetext{
${ }^{1}$ Referidos al $12 \%$ de $\mathrm{CH}$

${ }^{2}$ M1 y M2: muestreos constituidos por madera de árboles de 25 años del litoral, y de 15 años del suroeste respectivamente

${ }^{3}$ Valores medios. Entre paréntesis se indica desviación estándar

${ }^{4}$ Test Fisher LSD (Least Significant Difference). Dentro de cada muestreo, valores medios seguidos de igual letra en cada columna indica que no existen diferencias significativas $(\alpha=0,05)$
} 
Los bajos valores de rigidez observados especialmente en los grados G1 y G2 pueden ser atribuidos a la ocurrencia de madera juvenil, identificada entre otras características, por la presencia de médula en el 95\% de las vigas correspondientes a estos grados. En la madera juvenil es frecuente la ocurrencia de mayores ángulos microfibriles en la pared celular S2 y su incidencia negativa sobre el módulo elástico (Cave et al. 1994, Donaldson 2008). De acuerdo a lo observado en este estudio la madera juvenil tendría mayor influencia en la rigidez que en la resistencia, mientras que los nudos aparecen como el atributo de crecimiento con mayor incidencia sobre la resistencia de las piezas ensayadas.

La tabla 4 resume los resultados de los análisis estadísticos de las propiedades de compresión y tracción de los cuerpos de prueba de los muestreos M1 y M2. Los valores medios de resistencia de compresión paralela $\left(f_{c, 0}\right)$ de las piezas de M1 y M2 se situaron entre 19-26 MPa, y entre 17-23 MPa respectivamente, valores todos inferiores al reportado por Doyle et al. 1967, para madera aserrada 2" x 6" de pino del sur (Southern pine). Los valores medios de resistencia de tracción paralela $\left(f_{t, 0}\right)$ de las piezas de $\mathrm{M} 1$ y M2 variaron entre 10-33 MPa, y entre 9-17 MPa, respectivamente. Los resultados del grado superior de M1 son mayores a los observados por Doyle et al. (1966), para piezas de pino del sur, y junto con los del grado superior de M2, concuerdan con el rango de valores reportado por Kretschmann et al. (1992), para piezas de madera provenientes de árboles de 28 años de Pinus taeda. En las piezas del grado superior de M1 se observó valores de tensión de tracción mayores a los de compresión, contradiciendo lo comúnmente aceptado para piezas de tamaño estructural (Bowyer et al. 2003). Este comportamiento puede ser atribuido a la ausencia de nudosidades y las consecuentes leves distorsiones de la fibra, o presencia de pequeños nudos (de diámetros menores a $15 \mathrm{~mm}$ ) en las piezas que registraron los valores de resistencia de tracción más elevados. Dentro de cada muestreo, las tensiones $f_{c, 0} \mathrm{y} f_{t, 0}$ fueron analizadas estadísticamente comprobándose diferencias significativas entre las piezas de los tres grados, para ambos muestreos.

Tabla 4. Análisis estadístico de resistencias de compresión y tracción paralela a la fibra ${ }^{1}$.

\begin{tabular}{ccccc|ccc}
\hline & & \multicolumn{3}{c|}{ Compresión } & \multicolumn{3}{c}{ Tracción } \\
\cline { 3 - 8 } Muestreo $^{2}$ & Grado & $\begin{array}{c}\mathrm{N}^{\circ} \\
\text { rep }\end{array}$ & $\begin{array}{c}f_{c, 0}^{3} \\
{[\mathrm{MPa}]}\end{array}$ & LSD $^{4}$ & $\begin{array}{c}\mathrm{N}^{\circ} \\
\text { rep }\end{array}$ & $f_{\text {t, }}{ }^{3}[\mathrm{MPa}]$ & LSD $^{4}$ \\
\hline \multirow{3}{*}{ M1 } & GS & 91 & $26(4)$ & $\mathrm{a}$ & 89 & $33(12)$ & $\mathrm{a}$ \\
& G1 & 36 & $23(4)$ & $\mathrm{b}$ & 31 & $15(4)$ & $\mathrm{b}$ \\
& G2 & 22 & $19(4)$ & $\mathrm{c}$ & 35 & $10(4)$ & $\mathrm{c}$ \\
\hline \multirow{3}{*}{ M2 } & GS & 20 & $23(4)$ & $\mathrm{a}$ & 33 & $17(6)$ & $\mathrm{a}$ \\
& G1 & 55 & $20(3)$ & $\mathrm{b}$ & 50 & $12(4)$ & $\mathrm{b}$ \\
& G2 & 46 & $17(3)$ & $\mathrm{c}$ & 34 & $9(3)$ & $\mathrm{c}$ \\
\hline
\end{tabular}

${ }^{1}$ Referidos al $12 \%$ de CH

${ }^{2}$ M1 y M2: muestreos constituidos por madera de árboles de 25 años del litoral, y de 15 años del suroeste respectivamente

${ }^{3}$ Valores medios. Entre paréntesis se indica desviación estándar.

${ }^{4}$ Test Fisher LSD (Least Significant Difference). Dentro de cada muestreo, valores medios seguidos de igual letra en cada columna indica que no existen diferencias significativas $(\alpha=0,05)$. 


\section{Agrupamiento de datos y asignación a grados}

Como los valores de rigidez y densidad de los cuerpos de prueba de G1 y G2 del muestreo M1 fueron similares, se decidió agruparlos en una nueva categoría E5, refiriendo al valor medio del módulo de elasticidad global en flexión, y consecuentemente denominar a las piezas GS como E7. Análogamente, las piezas GS y G1 de M2 fueron agrupadas en E5 y las G2 como R.

Las tablas 5 y 6 resumen los valores medios y característicos de las propiedades mecánicas y de la densidad de los cuerpos de prueba de tamaño estructural.

Tabla 5. Propiedades de flexión y densidad ${ }^{1}$ de cuerpos de prueba de tamaño estructural de Pinus elliottii y P. taeda.

\begin{tabular}{ccccccccc}
\hline Muestreo $^{2}$ & Grado & $\begin{array}{c}\mathrm{N}^{\mathbf{2}} \\
\text { rep }\end{array}$ & $\begin{array}{c}E_{m, g}{ }^{3} \\
{[\mathrm{MPa}]}\end{array}$ & $\begin{array}{c}E_{m, l}{ }^{3} \\
{[\mathrm{MPa}]}\end{array}$ & $\begin{array}{c}f_{m}{ }^{3} \\
{[\mathrm{MPa}]}\end{array}$ & $\begin{array}{c}f_{m, k}{ }^{4} \\
{[\mathrm{MPa}]}\end{array}$ & $r^{3}\left[\mathrm{~kg} / \mathrm{m}^{3}\right]$ & $\begin{array}{c}r_{k}{ }^{4} \\
{\left[\mathrm{~kg} / \mathrm{m}^{3}\right]}\end{array}$ \\
\hline \multirow{2}{*}{$\mathrm{M} 1$} & E7 & 55 & 7489 & 7852 & 47 & 24 & 470 & 410 \\
& E5 & 47 & 5822 & 6107 & 28 & 13 & 419 & 341 \\
\hline \multirow{2}{*}{$\mathrm{M} 2$} & E5 & 105 & 5158 & 5414 & 27 & 15 & 373 & 320 \\
& $\mathrm{R}$ & 44 & 4681 & 4915 & 20 & 6 & 361 & 320 \\
\hline
\end{tabular}

${ }^{1}$ Referidos al $12 \%$ de $\mathrm{CH}$

${ }^{2} \mathrm{M} 1$ y M2: muestreos constituidos por madera de árboles de 25 años del litoral, y de 15 años del suroeste respectivamente

${ }^{3}$ Valores medios

${ }^{4}$ Valores característicos

Tabla 6. Propiedades de compresión y tracción paralela a la fibra ${ }^{1}$ de cuerpos de prueba de tamaño estructural de Pinus elliottii y P. taeda.

\begin{tabular}{ccccc|ccc}
\hline Muestreo $^{2}$ & Grupo & $\begin{array}{c}\mathrm{N}^{\mathbf{0}} \\
\text { rep }\end{array}$ & $\begin{array}{c}f_{c, 0}{ }^{3} \\
{[\mathrm{MPa}]}\end{array}$ & $\begin{array}{c}f_{c, 0, k}{ }^{4} \\
{[\mathrm{MPa}]}\end{array}$ & $\begin{array}{c}\mathrm{N}^{0} \\
\text { rep }\end{array}$ & $\begin{array}{c}f_{t, 0}{ }^{3} \\
{[\mathrm{MPa}]}\end{array}$ & $\begin{array}{c}f_{b 0, k}{ }^{4} \\
{[\mathrm{MPa}]}\end{array}$ \\
\hline \multirow{2}{*}{$\mathrm{M} 1$} & E7 & 91 & 26 & 20 & 89 & 33 & 14 \\
& E5 & 58 & 22 & 13 & 66 & 12 & 6 \\
\hline \multirow{2}{*}{$\mathrm{M} 2$} & E5 & 75 & 21 & 16 & 83 & 15 & 8 \\
& $\mathrm{R}$ & 46 & 17 & 11 & 34 & 9 & 4 \\
\hline
\end{tabular}

${ }^{1}$ Referidos al $12 \%$ de $\mathrm{CH}$

${ }^{2} \mathrm{M} 1$ y M2: muestreos constituidos por madera de árboles de 25 años del litoral, y de 15 años del suroeste respectivamente

${ }^{3}$ Valores medios

${ }^{4}$ Valores característicos

De acuerdo a EN 338 (2010) la asignación a una clase resistente queda determinada por el valor medio del módulo de elasticidad local $\left(E_{m, l}\right)$, el valor característico de la densidad $\left(\rho_{\mathrm{k}}\right)$, ambos corregidos al $12 \%$ de $\mathrm{CH}$, y el valor característico de la resistencia de flexión $\left(f_{m, k}\right)$ referido al $\mathrm{CH}$ en el momento de ensayo según lo indicado en EN 408 (2010). Para la clase C14 los valores correspondientes son $7000 \mathrm{MPa}, 14$ MPa y $290 \mathrm{~kg} / \mathrm{m}^{3}$. En forma similar, NCh 1198 (2006) establece para la madera de pino radiata grado $\mathrm{G} 2$, valores medios de módulo de elasticidad global $\left(E_{m, g}\right)$ y de resistencia característica de flexión, que ajustados para piezas de $150 \mathrm{~mm}$ de altura, alcanzan $8082 \mathrm{MPa}$ y $10,21 \mathrm{MPa}$, respectivamente, y valores de densidad característica de $370 \mathrm{~kg} / \mathrm{m}^{3}$. En ambas normas, la asignación a una clase resistente queda determinada por el valor más bajo de las tres propiedades al efectuar la comparación con los correspondientes valores exigidos por las normas, que en el presente estudio corresponde al del módulo de elasticidad. Comparando con los límites establecidos para la clase o grado estructural en cada norma, solamente el grupo E7 cumple con EN 338, mientras que ninguno con NCh 1198. 
Sin embargo, en concordancia con el estudio de simulación para pino radiata (CORMA 1990, citado en Wagner 1994) que definió un grado estructural para vigas de $45 \times 190 \mathrm{~mm}\left(\operatorname{con} f_{m, k}=5,0 \mathrm{MPa}\right.$ y $E_{m, g}=$ $5660 \mathrm{MPa})$ y otro para pie derechos de $45 \times 95 \mathrm{~mm}\left(\operatorname{con} f_{m, k}=4,0 \mathrm{MPa}\right)$, los resultados del presente trabajo podrían servir de base para la definición de un sistema de clases resistentes para madera de pinos uruguayos en el cual E5 sea admitido sólo para pie derechos y represente el límite inferior del rango.

Si bien las piezas de los grados superiores de M2 cuentan con propiedades mecánicas que permiten incluirlas en el grupo E5, no se recomienda su uso estructural y tampoco como pie derechos, ya que la mayoría de las piezas están constituidas en su totalidad por madera juvenil que posiblemente afecte su estabilidad dimensional y durabilidad.

Las piezas calificadas $\mathrm{R}$ tienen propiedades que son todas inferiores a las de referencia, por tanto son rechazadas.

Las figuras 1 a 4 representan las frecuencias acumuladas para el módulo de elasticidad y las resistencias de flexión, compresión y tracción paralela a la fibra de los cuerpos de prueba de madera de Pinus elliottii y $P$. taeda de 25 años provenientes del litoral. Se observa que el $61 \%$ de las piezas E7 cumple con los requisitos de rigidez establecidos en EN 338 para la clase C14, mientras que el porcentaje decrece a 25 en las piezas E5 (Figura 1a).

Al comparar con los valores de NCh 1198 para piezas de $150 \mathrm{~mm}$ de altura, se observa que el 40\% de las piezas E7 y el 15\% de las E5 cumplen con las exigencias de rigidez del grado G2 (Figura 1b).
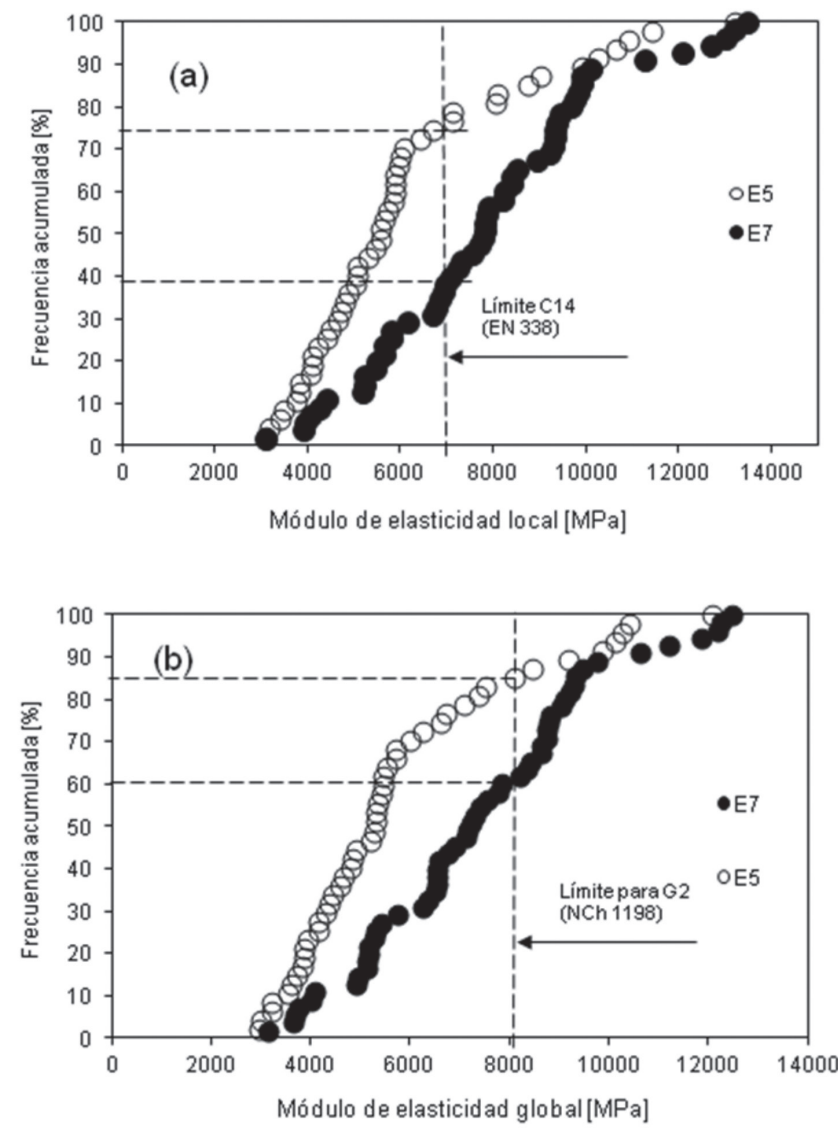

Figura 1. Frecuencia acumulada de: (a) módulo de elasticidad local y (b) módulo de elasticidad global para piezas clasificadas E7 y E5. 
El gráfico de frecuencia acumulada para la resistencia de flexión muestra que el 99 y $90 \%$ de las vigas E7 y E5, respectivamente, cumplen con los requisitos de la clase C14 de EN 338. Por otra parte, se observa que prácticamente la totalidad de las piezas E7 y E5 cumple con las exigencias del grado G2 de NCh 1198 (Figura 2). Cabe mencionar que la presentación de datos experimentales y la comparación con los umbrales de EN 338, y de NCh 1198, en un mismo gráfico (Figura 2), fue posible ya que el eventual ajuste por $\mathrm{CH}$ establecido en la norma chilena, no afectó sustancialmente los resultados experimentales (cercanos al 12\% al momento de ensayo, y que fuera mencionado anteriormente). Esta aclaración es válida también para las resistencias de compresión paralela y tracción paralela a la fibra, y se aplica a los datos representados en las Figuras 3 y 4.

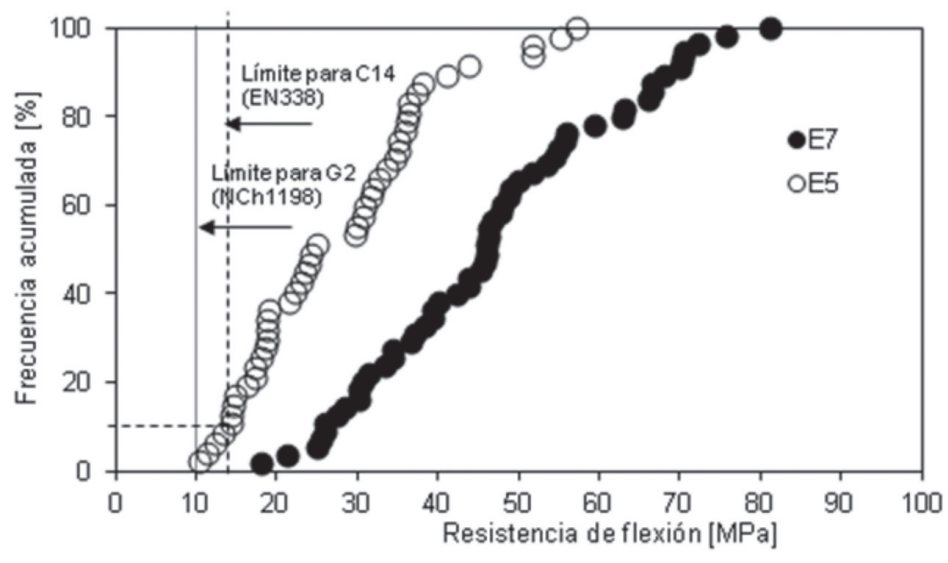

Figura 2. Frecuencia acumulada de resistencia de flexión para piezas clasificadas E7 y E5.

Los valores de resistencias de compresión paralela del 100 y del 90\% de las piezas E7 y E5 respectivamente, superan el umbral mínimo de $16 \mathrm{MPa}$ de EN 338. Esta observación se confirma para la totalidad de las piezas E7 y E5 que sobrepasan el límite ajustado para piezas de $150 \mathrm{~mm}$ de altura, de $11 \mathrm{MPa}$ de NCh 1198 (Figura 3).

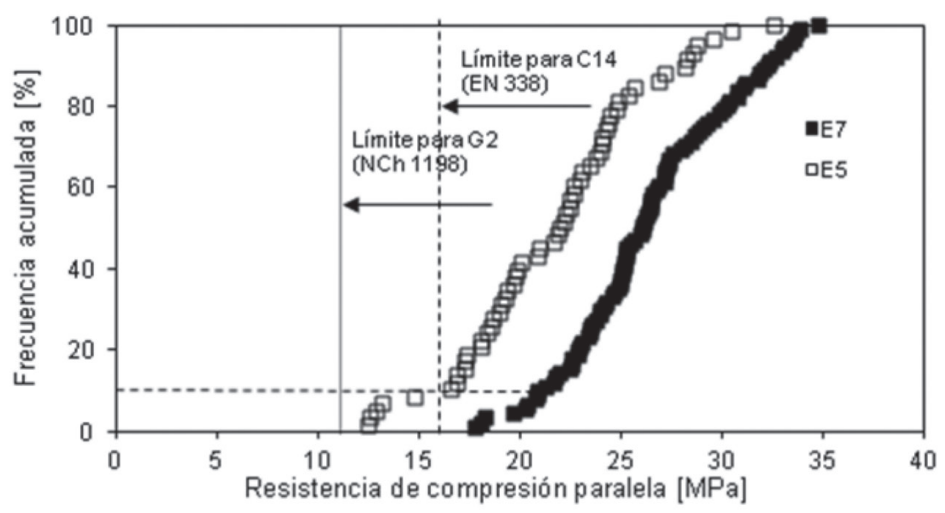

Figura 3. Frecuencia acumulada de resistencia de compresión paralela a la fibra para piezas clasificadas E7 y E5. 
Los valores de resistencias de tracción paralela del 98 y 82\% de las piezas E7 y E5 respectivamente, cumplen con los requisitos de la clase C14 de EN 338; estos porcentajes se incrementan hasta llegar a 100 y 85 para las piezas E7 y E5 respectivamente, cuando son comparados con los requeridos para el grado G2 de NCh 1198 (Figura 4).

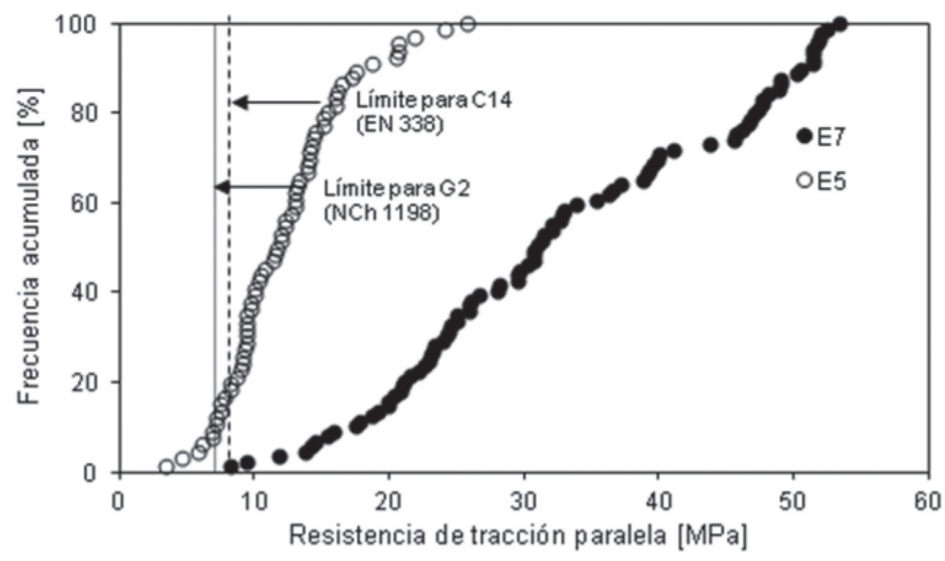

Figura 4. Frecuencia acumulada de resistencia de tracción paralela a la fibra para piezas clasificadas E7 y E5. 


\section{CONCLUSIONES}

Los resultados del presente estudio permiten establecer las siguientes conclusiones:

La aplicación de la norma de clasificación visual NCh 1207 a piezas de madera aserrada de pinos uruguayos, y la consiguiente asignación a un grado de calidad con una familia de propiedades estructurales asociada, determinó un porcentaje elevado de piezas rechazadas.

Los resultados de los ensayos en el grado fueron comparados con los valores de las propiedades físicas y mecánicas para las clases resistentes de EN 338 y los grados estructurales de NCh 1198. Basado en los límites de rigidez, resistencia de flexión y densidad de EN 338, solamente las piezas provenientes de árboles de 25 años del litoral del grupo superior (E7) pudieron ser asignadas a la clase inferior, C14, del sistema europeo. Al comparar con los límites de NCh 1198 para madera de pino radiata, se comprobó que ningún grupo cumplió con las exigencias del grado menor, G2, del sistema chileno. En ambos sistemas, la calificación quedó determinada por el valor más bajo de las tres propiedades al efectuar la comparación con los correspondientes valores exigidos por las normas, que en este estudio correspondió al del módulo de elasticidad.

Las piezas provenientes de árboles de 25 años del litoral del grupo inferior (E5) presentaron valores de rigidez menores a los exigidos en las normas EN 338 y NCh 1198. Sin embargo, los valores de resistencias y densidad observados fueron iguales o mayores a los de C14 o G2, lo cual permitiría admitir su uso como pie derechos (de 45 x $95 \mathrm{~mm}$ ) de tabiquerías en estructuras de entramados ligeros.

Las propiedades de las piezas de madera de árboles de 15 años del suroeste no cumplen con los requisitos mínimos para uso estructural. Se sugiere no utilizar esta madera en componentes estructurales y se recomienda a la industria que la procese con otros fines.

Los resultados del presente estudio podrán servir de base para la definición de un sistema de clases resistentes para madera de pinos cultivados en Uruguay.

\section{AGRADECIMIENTOS}

Los autores agradecen a la Agencia Nacional de Investigación e Innovación (ANII), Uruguay, Fondo María Viñas- PR-FMV-2009-1-2772 por los fondos para este proyecto. 


\section{REFERENCIAS}

ASTM International. 2011. Standard test methods of static tests of lumber in structural sizes. ASTM D198-09. ASTM, West Conshohocken, Pennsylvania.

ASTM International. 2011. Standard practice for establishing allowable properties for visually graded dimension lumber from in-grade test of full-size specimens. ASTM D1990-07. ASTM, West Conshohocken, Pennsylvania.

ASTM International. 2011. Standard test methods for specific gravity of wood and wood-based materials. ASTM D 2395-07. West Conshohocken, Pennsylvania.

ASTM International. 2011. Standard practice for sampling and data-analysis for structural wood and wood-based panels. ASTM D2915-10. West Conshohocken, Pennsylvania.

Biblis, E.J. 2006. Flexural properties and compliance to visual grade requirements of 2 by 4 and 2 by 6 loblolly pine lumber obtained from a 19-year-old plantation. Forest Prod J 56(9):71-73.

Bodig, J.; Jayne, B.A. 1993. Mechanics of wood and wood composites, Krieger Publishing Co, Malabar, FL, $712 \mathrm{pp}$.

Bowyer, J.L.; Shmulsky, R.; Haygreen, J.G. 2003. Forest products and wood science: An introduction. $4^{\text {th }}$ ed. Blackwell Publishing, Oxford, UK, 564 pp.

Cave, I.D.; Walker, J.C.F. 1994. Stiffness of wood in fast-grown plantation softwood: the influcence of microfibril angle. Forest Prod J 44(5):43-48.

Comité Européen de Normalisation. 2010. Structural timber-strength classes. EN 338. CEN, Brussels.

Comité Européen de Normalisation. 2010. Structural timber: determination of characteristic values of mechanical properties and density. EN 384. CEN, Brussels.

Comité Européen de Normalisation. 2010. Structural timber: determination of characteristic values of mechanical properties and density. EN 384. CEN, Brussels.

Comité Européen de Normalisation. 2010. Timber structure-structural timber and glued laminated timber-determination of some physical and mechanical properties. EN 408. CEN, Brussels.

Corporación Chilena de la Madera. 1990. Proyecto Grados estructurales de pino radiata. Gerencia de Proyectos. CORMA, Santiago.

Donaldson, L.A. 2008. Microfibril angle: Measurement, variation and relationship- A review. IAWA J 29(4): 345-386.

Doyle, D.V.; Markwardt, L.J. 1966. Properties of southern pine in relation to strength grading of dimension lumber. Res. Pap. FPL 64. U.S. Department of Agriculture, Forest Service, Forest Products Laboratory, Madison WI.

Doyle, D.V.; Markwardt, L.J. 1967. Tension parallel-to-grain properties of southern pine dimension lumber. Res. Pap. FPL 84. U.S. Department of Agriculture, Forest Service, Forest Products Laboratory, Madison WI. 
Instituto Nacional de Normalización. 1990. Pino radiata. Clasificación visual para uso estructural. Especificaciones de los grados de calidad. NCh 1207-Of 1990. INN, Santiago.

Instituto Nacional de Normalización. 2005. Pino radiata. Clasificación visual para uso estructural. Especificaciones de los grados de calidad. NCh 1207-Of 2005. INN, Santiago.

Instituto Nacional de Normalización. 2006. Madera - Construcciones en madera- Cálculo. NCh 1198. INN, Santiago.

Instituto Nacional de Normalización. 2006. Madera estructural. Determinación de propiedades físicas y mecánicas de la madera clasificada por su resistencia. Parte 1: Métodos de ensayo en tamaño estructural. NCh 3028/1-Of 2006. INN, Santiago.

Instituto Nacional de Normalización.NCh. 2006. Madera estructural. Determinación de propiedades físicas y mecánicas de la madera clasificada por su resistencia. Parte 2: Muestreo y evaluación de los valores característicos de piezas en tamaño estructural. NCh 3028/2-Of 2008. INN, Santiago.

Kretschmann, D.E.; Bendtsen, B.A.1992. Ultimate tensile stress and modulus of elasticity of fastgrown plantation loblolly pine lumber. Wood Fiber Sci 24(2):189-203.

Ministerio de Agricultura, Ganadería Y Pesca. 2005. Boletín estadístico. Dirección General Forestal, MGAP. 44 pp.

Ministerio de Agricultura, Ganadería y Pesca. 2010. Monitoreo de los recursos forestales. Inventario forestal nacional. Resumen de resultados. Dirección General Forestal, MGAP.

Ministerio de Vivienda, Ordenamiento Territorial y Medio Ambiente. 2011. Sistemas constructivos no tradicionales. MVOTMA.

Ministry of Agriculture, Forestry And Fisheries. 1991. Japanese agricultural standard for structural softwood lumber. JAS 143. Tokyo.

Montevideo Portal. 2013. [Disponible en línea]<http://www.uruguaysustentable.com.uy/politicaeconomia/mvotma-trabaja-en-seis-lineas-para-resolver-problemas-de-vivienda-en-uruguay/>. [accesado febrero 2014].

Moya, L.; Laguarda, M.F.; Cagno, M.; Cardoso, A.; Gatto, F.; O'Neil, H. 2013. Physical and mechanical properties of loblolly and slash pine wood from Uruguayan plantations. Forest Prod $J$ 63(3-4):128-137.

O’Neill, H.; Tarigo, F.; Trambauer, C. 2002. Propiedades mecánicas de Pinus elliottii Eng. del litoral de Uruguay. Informe $\mathrm{N}^{\circ} 1$. Grupo Técnico de Madera Aserrada de Pino GT3. Laboratorio Tecnológico del Uruguay, Montevideo, $41 \mathrm{pp}$.

O’Neill, H.; Tarigo, F.; Trambauer, C. 2002. Propiedades mecánicas de Pinus taeda L. del litoral de Uruguay. Informe $\mathrm{N}^{\circ}$ 2. Grupo Técnico de Madera Aserrada de Pino GT3. Laboratorio Tecnológico del Uruguay, Montevideo, $47 \mathrm{pp}$.

O'Neill, H.; Tarigo, F. 2003. Comportamiento en flexión de E. grandis, P. taeda y P. elliottii de madera de tamaño real y de pequeñas probetas sin defectos. Nota técnica. Laboratorio Tecnológico del Uruguay, Montevideo, 6 pp. 
$\mathbf{O}^{\prime}$ Neill, H. 2004. Estimación de la calidad de la madera producida en el Uruguay para uso estructural y su evaluación en servicio por métodos no destructivos. Nota técnica. Laboratorio Tecnológico del Uruguay, Montevideo, 9 pp.

Pérez Del Castillo, A. 2001. Módulo de elasticidad y módulo de rotura en tablas de tamaño real de Pinus elliottii del sur de Uruguay. Informe de investigación N ${ }^{\circ} 7$. Laboratorio Tecnológico del Uruguay, Montevideo, $29 \mathrm{pp}$.

Pérez Del Castillo, A.; Venturino, A. 2003. Inspección visual de tablas de tamaño real de Eucalyptus grandis, Pinus taeda y Pinus elliottii de diferentes sitios del Uruguay. Informe de investigación $\mathrm{N}^{\circ} 15$. Laboratorio Tecnológico del Uruguay, Montevideo, 28 pp.

Pérez Favaro, A.; De Castro, R.; Otha, S. 2000. Ensayos de propiedades mecánicas de Pinus taeda por seis métodos no destructivos. Informe de investigación $\mathrm{N}^{\circ} 1$. Laboratorio Tecnológico del Uruguay, Montevideo, $24 \mathrm{pp}$.

USDA. 1999. Wood Handbook: Wood as an engineering material. Gen. Tech. Rep. FPL-GTR-113. USDA For. Serv. Forest Products Laboratory, Madison, WI. 463 pp.

Wagner, M. 1994. Clasificación de la madera estructural. Notas de clase. Diplomado en Diseño y construcción en madera. Universidad del Bío-Bío, Concepción. 14 pp. 